\title{
Study of an implicit type coupled system of fractional differential equations by means of topological degree theory
}

\author{
Muhammad Sarwar ${ }^{1 *}$, Anwar Ali ${ }^{1}$, Mian Bahadur Zada ${ }^{1}$, Hijaz Ahmad ${ }^{2,3 *}$ and Taher A. Nofal ${ }^{4}$
}

\author{
"Correspondence: \\ sarwarswati@gmail.com; \\ hijaz555@gmail.com \\ 'Department of Mathematics, \\ University of Malakand, Chakadara \\ Dir(L), Khyber Pakhtunkhwa, \\ Pakistan \\ ${ }^{2}$ Department of Basic Sciences, \\ University of Engineering and \\ Technology, Peshawar 25000, \\ Pakistan \\ Full list of author information is \\ available at the end of the article
}

\begin{abstract}
In this work, a sufficient condition required for the presence of positive solutions to a coupled system of fractional nonlinear differential equations of implicit type is studied. To study sufficient conditions essential for the existence of unique solution degree theory is used. Two examples are given to illustrate the established results.
\end{abstract}

MSC: 34A08; 35R11

Keywords: Differential equations of fractional order; Integral boundary value problem; Topological degree theory

\section{Introduction}

The concept of fractional differential equations (abbreviated as FDEs) has been examined and considered seeing its usefulness and plentiful presentations in different disciplines of applied science, engineering, and technology such as computer networking, fluid dynamics, control theory, mathematical biology, economics, viscoelasticity, optimization theory, and control theory [1-8]. Nonlinear fractal oscillator is recognized in a fractal space by fractal derivative, and its variational principle is gained for a thin film equation [9]. In a fractal space He's fractional derivative [10] is assumed to originate evolution equations involving fractional order [11]. In a fractal process, the Fornberg-Whitham fractional equation through He's fractional derivative is considered [12], and future challenges of fractal calculus have been illustrated from two-scale thermodynamics to fractal variational principle by Ji-Huan He [13]. Substantial consideration has been given to the presence of solutions of initial and boundary value problems (BVPs) having CFD.

Diverse sort of problems dedicated to FDEs, like local and nonlocal BVPs, Dirichlet and Neumann BVPs, integral BVPs, and impulsive BVPs, have been explored so far. An indispensable class of FDEs named implicit fractional differential equations (shortly IFDEs) has been considered by numerous writers. This is because of the point that many problems of finances and decision-making can be modeled by using IFDEs. Recently more courtesy has been given to scrutinizing sufficient conditions essential for the existence of solutions to IFDEs. It was observed sensibly that the existence of solutions to IFDEs had a lot of solicitations in optimization theory, quantitative theory, viscoelasticity, and fluid mechanics

(c) The Author(s) 2021. This article is licensed under a Creative Commons Attribution 4.0 International License, which permits use sharing, adaptation, distribution and reproduction in any medium or format, as long as you give appropriate credit to the original author(s) and the source, provide a link to the Creative Commons licence, and indicate if changes were made. The images or other third party material in this article are included in the article's Creative Commons licence, unless indicated otherwise in a credit line to the material. If material is not included in the article's Creative Commons licence and your intended use is not permitted by statutory regulation or exceeds the permitted use, you will need to obtain permission directly from the copyright holder. To view a copy of this licence, visit http://creativecommons.org/licenses/by/4.0/. 
[14-19]. Nonlocal Cauchy problem via a fractional operator including power kernel in Banach spaces was considered in [20]. The fractional hampered generalized regularized long wave equation in the sagacity of Caputo, Atangana-Baleanu, and Caputo-Fabrizio fractional derivatives was investigated in [21]. In [22] authors presented a method for nonlinear fractional regularized long-wave (RLW) models. Mehmet Yavuz [23] inspected innovative solutions of fractional order best valuing models and their fundamental mathematical studies.

Fixed point concept has been used to probe the existence and uniqueness for some problems. Operating these notions, one needs strong compact settings due to which the area is limited to some BVPs. To spread the methods to additional classes of BVPs, mathematicians have been attracted to finding a tool of nonlinear analysis. One of the strong tools is the degree method. After studying the present literature, we pointed out that IFDEs having integral boundary conditions have not been properly studied by the degree method. There are very few results in the literature which utilized the degree method for the existence of solutions to initial and some BVPs having CFD [1, 24-27]. Therefore, inspired by the applications of IFDEs, Samina et al. [28] investigated the presence of solutions to the following coupled system "of IFDEs through fixed point theory

$$
\left\{\begin{array}{l}
D^{\kappa} \mathrm{u}(\ell)=\mathcal{F}\left(\ell, \mathrm{w}(\ell), D^{\kappa} \mathrm{u}(\ell)\right), \\
D^{\delta} \mathrm{w}(\ell)=\overline{\mathcal{F}}\left(\ell, \mathrm{u}(\ell), D^{\delta} \mathrm{w}(\ell)\right), \\
\mathrm{u}(0)=-\mathrm{u}(\xi), \quad \mathrm{u}^{\prime}(0)=-\mathrm{u}^{\prime}(\xi), \\
\mathrm{w}(0)=-\mathrm{w}(\xi), \quad \mathrm{w}^{\prime}(0)=-\mathrm{w}^{\prime}(\xi),
\end{array}\right.
$$

where $\kappa, \delta \in(1,2], \xi \in(0, \infty), \ell \in[0, \xi]$ and $\mathcal{F}, \overline{\mathcal{F}}: \mathfrak{J} \times \mathfrak{R} \times \mathfrak{R} \rightarrow \mathfrak{R}$ are nonlinear continuous functions." Using fixed point theory, Cabada et al. [29] discussed the following problem:

$$
\begin{aligned}
& D^{\kappa} \mathrm{u}(\ell)+\mathcal{F}(\ell, \mathrm{u}(\ell))=0, \quad \ell \in(0,1), \\
& \mathrm{u}(0)+\mathrm{u}^{\prime \prime}(0)=0, \quad \mathrm{u}(1)=\zeta \int_{0}^{1} \mathrm{u}(\mathrm{s}) d \mathrm{~s},
\end{aligned}
$$

where $2<\kappa<3,0<\zeta<2, D$ is the CFD and $\mathcal{F}: \mathfrak{J} \times[0, \infty) \rightarrow[0, \infty)$.

Motivated by [28] and [29], we use degree theory and investigate some suitable conditions for uniqueness and existence of solutions to the following IFDEs:

$$
\begin{cases}D^{\kappa} \mathrm{u}(\ell)=\mathcal{F}\left(\ell, \mathrm{w}(\ell), D^{\kappa} \mathrm{u}(\ell)\right), & \ell \in \mathfrak{J}, \\ D^{\delta} \mathrm{w}(\ell)=\overline{\mathcal{F}}\left(\ell, \mathrm{u}(\ell), D^{\delta} \mathrm{w}(\ell)\right), & \ell \in \mathfrak{J}, \\ \mathrm{u}(0)=r(\mathrm{u}), \quad \mathrm{u}^{\prime}(0)=\mathrm{u}_{o}, & \mathrm{u}(1)=\frac{1}{\Gamma(\kappa)} \int_{0}^{1}(1-\mathrm{s})^{\kappa-1} \mathrm{~g}_{1}(\mathrm{~s}, \mathrm{u}(\mathrm{s})) d \mathrm{~s}, \\ \mathrm{w}(0)=h(\mathrm{w}), \quad \mathrm{w}^{\prime}(0)=\mathrm{w}_{o}, \quad \mathrm{w}(1)=\frac{1}{\Gamma(\delta)} \int_{0}^{1}(1-\mathrm{s})^{\delta-1} \mathrm{~g}_{2}(\mathrm{~s}, \mathrm{w}(\mathrm{s})) d \mathrm{~s},\end{cases}
$$

where $\kappa, \delta \in(2,3], D$ denotes the CFD, $\mathcal{F}, \overline{\mathcal{F}}: \mathfrak{J} \times \mathfrak{R} \times \mathfrak{R} \rightarrow \mathfrak{R}, \mathrm{g}_{1}, \mathrm{~g}_{2}: \mathfrak{J} \times \mathfrak{R} \rightarrow \mathfrak{R}$, and $r, h: \mathfrak{J} \rightarrow \mathfrak{R}$ are continuous functions.

\section{Preliminaries}

To prove the main results, we need some definitions and results in the sequel from the existing literature. Throughout the work the notations $\mathcal{M}=C(\mathfrak{J}, \mathfrak{R})$ and $\mathcal{N}=C(\mathfrak{J}, \mathfrak{R})$ are 
used for Banach spaces having the norm $\|\mathrm{u}\|=\sup \{|\mathrm{u}(\ell)|: \ell \in \mathfrak{J}\}$. The product $\mathcal{M} \times \mathcal{N}$ is a Banach space with the norm $\|(\mathrm{u}, \mathrm{w})\|=\|\mathrm{u}\|+\|\mathrm{w}\|$.

Definition 2.1 ([30]) "Let $\mathcal{W}: \mathcal{V} \rightarrow \mathcal{M}$ be a bounded continuous function, where $\mathcal{V} \subseteq \mathcal{M}$. Then, for all bounded subset $S \subseteq \mathcal{V}, \mathcal{W}$ is

(1) $\sigma$-Lipschitz if $\exists \mathcal{K} \geq 0 \ni \sigma(\mathcal{W}(S)) \leq \mathcal{K} \sigma(S), \forall$ bounded subsets $S \subseteq \mathcal{V}$;

(2) strict $\sigma$-contraction if $\exists 0 \leq \mathcal{K}<1$ with $\sigma(\mathcal{W}(S)) \leq \mathcal{K} \sigma(S), \forall$ bounded sets $S \subseteq \mathcal{V}$;

(3) $\sigma$-condensing if $\sigma(\mathcal{W}(S))<\sigma(S), \forall$ bounded sets $S \subseteq \mathcal{V}$ having $\sigma(S)>0$. In the other sense, $\sigma(\mathcal{W}(S)) \geq \sigma(S)$ implies $\sigma(S)=0$.

Furthermore, $\mathcal{W}: \mathcal{V} \rightarrow \mathcal{M}$ is Lipschitz whenever $\exists \mathcal{K}>0$ provided

$$
\|\mathcal{W}(\mathrm{u})-\mathcal{W}(\mathrm{w})\| \leq \mathcal{K}|\mathrm{u}-\mathrm{w}|, \quad \forall \mathrm{u}, \mathrm{w} \in \mathcal{V}
$$

Further, if $\mathcal{K}<1$, then $\mathcal{W}$ is a strict contraction."

Proposition 2.1 ([31]) If $\mathcal{W}, T: \mathcal{V} \rightarrow \mathcal{M}$ are $\sigma$-Lipschitz maps having constants $\mathcal{K}_{1}$ and $\mathcal{K}_{2}$ respectively, then $\mathcal{W}+T$ is $\sigma$-Lipschitz having constant $\mathcal{K}_{1}+\mathcal{K}_{2}$.

Proposition 2.2 ([31]) If $\mathcal{W}: \mathcal{V} \rightarrow \mathcal{M}$ is Lipschitz having constant $\mathcal{K}$, then $\mathcal{W}$ is $\sigma$ Lipschitz having the same constant $\mathcal{K}$.

Proposition 2.3 ([31]) If $\mathcal{W}: \mathcal{V} \rightarrow \mathcal{M}$ is compact, then $\mathcal{W}$ is $\sigma$-Lipschitz having the constant $\mathcal{K}=0$.

Theorem 2.1 ([31]) Let $\mathcal{W}: \mathcal{M} \rightarrow \mathcal{M}$ be $\sigma$-condensing having

$$
\Theta=\{\mathrm{u} \in \mathcal{M}: \exists 0 \leq \vartheta \leq 1 \text { with } \mathrm{u}=\vartheta \mathcal{W} \mathrm{u}\}
$$

If $\Theta$ is bounded in $\mathcal{M}$, so $\exists r>0 \ni \Theta \subset S_{r}(0)$, so the degree

$$
Q\left(I-\vartheta \mathcal{W}, S_{r}(0), 0\right)=1, \quad \forall \vartheta \in \mathfrak{J}
$$

It means that $\mathcal{W}$ has at least one fixed point.

Definition 2.2 ([32]) “The arbitrary order $(\kappa>0)$ integral of a function $\mathcal{F}: \mathfrak{R}^{+} \rightarrow \mathfrak{R}$ is given by

$$
I^{\kappa} \mathcal{F}(\ell)=\frac{1}{\Gamma(\kappa)} \int_{0}^{\ell}(\ell-\mathrm{s})^{\kappa-1} \mathcal{F}(\mathrm{s}) d \mathrm{~s}
$$

Definition 2.3 ([32]) The arbitrary order $(\kappa>0)$ derivative of a function $\mathcal{F}: \mathfrak{R}^{+} \rightarrow \mathfrak{R}$ in the Caputo sense is given by

$$
D^{\kappa} \mathcal{F}(\ell)=\frac{1}{\Gamma(n-\kappa)} \int_{0}^{\ell}(\ell-\mathrm{s})^{n-\kappa-1} \mathcal{F}^{(n)}(\mathrm{s}) d \mathrm{~s} . "
$$


Lemma 2.1 [32] Let $\kappa>0$, then

$$
I^{\kappa}\left[D^{\kappa} h(\ell)\right]=h(\ell)+c_{0}+c_{1} \ell+c_{2} \ell^{2}+\cdots+c_{n-1} \ell^{n-1}
$$

for arbitrary $c_{i} \in \mathfrak{R}, i=0,1,2, \ldots, n-1$.

\section{Main results}

Before studying the existence results for BVP (1.1), we list the following assumptions.

$\left(C_{1}\right)$ For random $\mathrm{u}, \mathrm{w}, \overline{\mathrm{u}}, \overline{\mathrm{w}} \in \mathfrak{R}, \exists$ numbers $k_{r}, k_{h} \in[0,1)$ with

$$
\begin{aligned}
& |r(\mathrm{u})-r(\overline{\mathrm{u}})| \leq k_{r}|\mathrm{u}-\overline{\mathrm{u}}|, \\
& |h(\mathrm{w})-h(\overline{\mathrm{w}})| \leq k_{h}|\mathrm{w}-\overline{\mathrm{w}}| .
\end{aligned}
$$

$\left(C_{2}\right)$ For arbitrary $\mathrm{u}, \mathrm{w} \in \mathfrak{R}, \exists$ constants $c_{r}, c_{h}, M_{r}, M_{h}$ with

$$
\begin{aligned}
& |r(\mathrm{u})| \leq c_{r}|\mathrm{u}|+M_{r}, \\
& |h(\mathrm{w})| \leq c_{h}|\mathrm{w}|+M_{h} .
\end{aligned}
$$

$\left(C_{3}\right)$ For arbitrary $\mathrm{u}, \mathrm{w} \in \mathfrak{R}, \exists$ constants $z_{\mathrm{g}_{1}}, z_{\mathrm{g}_{2}}, N_{\mathrm{g}_{1}}, N_{\mathrm{g}_{2}}$ with

$$
\begin{aligned}
& \left|\mathrm{g}_{1}(\mathrm{~s}, \mathrm{u}(\mathrm{s}))\right| \leq z_{\mathrm{g}_{1}}|\mathrm{u}|+N_{\mathrm{g}_{1}}, \\
& \left|\mathrm{~g}_{2}(\mathrm{~s}, \mathrm{w}(\mathrm{s}))\right| \leq z_{\mathrm{g}_{2}}|\mathrm{w}|+N_{\mathrm{g}_{2}} .
\end{aligned}
$$

$\left(C_{4}\right)$ For arbitrary $\mathrm{u}, \mathrm{w} \in \mathfrak{R}, \exists$ constants $c_{1}, d_{1}>0,0<c_{2}, d_{2}<1, M_{\mathcal{F}}, M_{\overline{\mathcal{F}}}$ with

$$
\begin{aligned}
& |\mathcal{F}(\mathrm{s}, \mathrm{w}(\mathrm{s}), \omega(\mathrm{s}))| \leq c_{1}|\mathrm{w}|+c_{2}|\omega|+M_{\mathcal{F}} \\
& |\overline{\mathcal{F}}(\mathrm{s}, \mathrm{u}(\mathrm{s}), z(\mathrm{~s}))| \leq d_{1}|\mathrm{u}|+d_{2}|z|+M_{\overline{\mathcal{F}}}
\end{aligned}
$$

where $D^{\kappa} \mathrm{u}(\mathrm{s})=\omega(\mathrm{s})$ and $D^{\kappa} \mathrm{w}(\mathrm{s})=z(\mathrm{~s})$.

$\left(C_{5}\right)$ For arbitrary $\mathrm{u}, \mathrm{w}, \overline{\mathrm{u}}, \overline{\mathrm{w}} \in \mathfrak{R}, \exists$ constants $a_{1}, a_{2}$ with

$$
\begin{aligned}
& \left|\mathrm{g}_{1}(\mathrm{~s}, \mathrm{u}(\mathrm{s}))-\mathrm{g}_{1}(\mathrm{~s}, \overline{\mathrm{u}}(\mathrm{s}))\right| \leq a_{1}|\mathrm{u}-\overline{\mathrm{u}}| \\
& \left|\mathrm{g}_{2}(\mathrm{~s}, \mathrm{w}(\mathrm{s}))-\mathrm{g}_{2}(\mathrm{~s}, \overline{\mathrm{w}}(\mathrm{s}))\right| \leq a_{2}|\mathrm{w}-\overline{\mathrm{w}}|
\end{aligned}
$$

$\left(C_{6}\right)$ For arbitrary u, w, $\overline{\mathrm{u}}, \overline{\mathrm{w}} \in \mathfrak{R}, \exists$ constants $C_{\mathrm{g}_{1}}, C_{\mathrm{g}_{2}}>0,0<D_{\mathrm{g}_{1}}, D_{\mathrm{g}_{2}}<1$ with

$$
\begin{aligned}
& |\mathcal{F}(\mathrm{s}, \mathrm{w}(\mathrm{s}), \omega(\mathrm{s}))-\mathcal{F}(\mathrm{s}, \overline{\mathrm{w}}(\mathrm{s}), \bar{\omega}(\mathrm{s}))| \leq C_{\mathrm{g}_{1}}|\mathrm{w}-\overline{\mathrm{w}}|+D_{\mathrm{g}_{1}}|\omega-\bar{\omega}|, \\
& |\overline{\mathcal{F}}(\mathrm{s}, \mathrm{u}(\mathrm{s}), z(\mathrm{~s}))-\overline{\mathcal{F}}(\mathrm{s}, \overline{\mathrm{u}}(\mathrm{s}), \bar{z}(\mathrm{~s}))| \leq C_{\mathrm{g}_{2}}|\mathrm{u}-\overline{\mathrm{u}}|+D_{\mathrm{g}_{2}}|z-\bar{z}|
\end{aligned}
$$

where $D^{\kappa} \mathrm{u}(\mathrm{s})=\omega(\mathrm{s})$ and $D^{\kappa} \mathrm{w}(\mathrm{s})=z(\mathrm{~s})$.

Lemma 3.1 Let the integrable function $h: \mathfrak{J} \rightarrow \mathfrak{R}$. Then the IFDE

$D^{\kappa} \mathrm{u}(\ell)=h(\ell), \quad 2<\kappa \leq 3$, 
with boundary condition of type

$$
\mathrm{u}(0)=r(\mathrm{u}), \quad \mathrm{u}^{\prime}(0)=\mathrm{u}_{o}, \quad \mathrm{u}(1)=\frac{1}{\Gamma(\kappa)} \int_{0}^{1}(1-\mathrm{s})^{\kappa-1} \mathrm{~g}_{1}(\mathrm{~s}, \mathrm{u}(\mathrm{s})) d \mathrm{~s},
$$

has a solution

$$
\begin{aligned}
\mathrm{u}(\ell)= & \left(1-\ell^{2}\right) r(\mathrm{u})+\left(\ell-\ell^{2}\right) \mathrm{u}_{o}+\frac{\ell^{2}}{\Gamma(\kappa)} \int_{0}^{1}(1-\mathrm{s})^{\kappa-1} \mathrm{~g}_{1}(\mathrm{~s}, \mathrm{u}(\mathrm{s})) d \mathrm{~s} \\
& +\frac{1}{\Gamma(\kappa)} \int_{0}^{\ell}(\ell-\mathrm{s})^{\kappa-1} h(\mathrm{~s}) d \mathrm{~s}-\frac{\ell^{2}}{\Gamma(\kappa)} \int_{0}^{1}(1-\mathrm{s})^{\kappa-1} h(\mathrm{~s}) d \mathrm{~s} .
\end{aligned}
$$

Proof Applying the operator $I^{\kappa}$ to $D^{\kappa} \mathrm{u}(\ell)=h(\ell)$, and by Lemma 2.1, we have

$$
\mathrm{u}(\ell)=c_{0}+c_{1} \ell+c_{2} \ell^{2}+I^{\kappa} h(\ell)
$$

Utilizing the boundary conditions to (3.1), we get

$$
c_{0}=r(\mathrm{u}), \quad c_{1}=\mathrm{u}_{o}, \quad c_{2}=-r(\mathrm{u})-\mathrm{u}_{o}-I^{\kappa} h(1)+\frac{1}{\Gamma(\kappa)} \int_{0}^{1}(1-\mathrm{s})^{\kappa-1} \mathrm{~g}_{1}(\mathrm{~s}, \mathrm{u}(\mathrm{s})) d \mathrm{~s} .
$$

Substituting in equation (3.1), we have

$$
\begin{aligned}
\mathrm{u}(\ell)= & r(\mathrm{u})+\mathrm{u}_{o} \ell-\ell^{2} r(\mathrm{u})-\ell^{2} \mathrm{u}_{o}+\frac{\ell^{2}}{\Gamma(\kappa)} \int_{0}^{1}(1-\mathrm{s})^{\kappa-1} \mathrm{~g}_{1}(\mathrm{~s}, \mathrm{u}(\mathrm{s})) d \mathrm{~s} \\
& -\frac{\ell^{2}}{\Gamma(\kappa)} \int_{0}^{1}(1-\mathrm{s})^{\kappa-1} h(\mathrm{~s}) d \mathrm{~s}+\frac{1}{\Gamma(\kappa)} \int_{0}^{\ell}(\ell-\mathrm{s})^{\kappa-1} h(\mathrm{~s}) d \mathrm{~s} \\
= & \left(1-\ell^{2}\right) r(\mathrm{u})+\left(\ell-\ell^{2}\right) \mathrm{u}_{o}+\frac{\ell^{2}}{\Gamma(\kappa)} \int_{0}^{1}(1-\mathrm{s})^{\kappa-1} \mathrm{~g}_{1}(\mathrm{~s}, \mathrm{u}(\mathrm{s})) d \mathrm{~s} \\
& -\frac{\ell^{2}}{\Gamma(\kappa)} \int_{0}^{1}(1-\mathrm{s})^{\kappa-1} \mathcal{F}\left(\mathrm{s}, \mathrm{w}(\mathrm{s}), D^{\kappa} \mathrm{u}(\mathrm{s})\right) d \mathrm{~s} \\
& +\frac{1}{\Gamma(\kappa)} \int_{0}^{\ell}(\ell-\mathrm{s})^{\kappa-1} \mathcal{F}\left(\mathrm{s}, \mathrm{w}(\mathrm{s}), D^{\kappa} \mathrm{u}(\mathrm{s})\right) d \mathrm{~s} .
\end{aligned}
$$

By Lemma 3.1, the solutions of coupled system (1.1) are solutions of the following system of integral equations:

$$
\left\{\begin{aligned}
\mathrm{u}(\ell)= & \left(1-\ell^{2}\right) r(\mathrm{u})+\left(\ell-\ell^{2}\right) \mathrm{u}_{o}+\frac{\ell^{2}}{\Gamma(\kappa)} \int_{0}^{1}(1-\mathrm{s})^{\kappa-1} \mathrm{~g}_{1}(\mathrm{~s}, \mathrm{u}(\mathrm{s})) d \mathrm{~s} \\
& -\frac{\ell^{2}}{\Gamma(\kappa)} \int_{0}^{1}(1-\mathrm{s})^{\kappa-1} \mathcal{F}\left(\mathrm{s}, \mathrm{w}(\mathrm{s}), D^{\kappa} \mathrm{u}(\mathrm{s})\right) d \mathrm{~s} \\
& +\frac{1}{\Gamma(\kappa)} \int_{0}^{\ell}(\ell-\mathrm{s})^{\kappa-1} \mathcal{F}\left(\mathrm{s}, \mathrm{w}(\mathrm{s}), D^{\kappa} \mathrm{u}(\mathrm{s})\right) d \mathrm{~s}, \\
\mathrm{w}(\ell)= & \left(1-\ell^{2}\right) h(\mathrm{w})+\left(\ell-\ell^{2}\right) \mathrm{w}_{o}+\frac{\ell^{2}}{\Gamma(\delta)} \int_{0}^{1}(1-\mathrm{s})^{\delta-1} \mathrm{~g}_{2}(\mathrm{~s}, \mathrm{w}(\mathrm{s})) d \mathrm{~s} \\
& -\frac{\ell^{2}}{\Gamma(\delta)} \int_{0}^{1}(1-\mathrm{s})^{\delta-1} \overline{\mathcal{F}}\left(\mathrm{s}, \mathrm{u}(\mathrm{s}), D^{\delta} \mathrm{w}(\mathrm{s})\right) d \mathrm{~s} \\
& +\frac{1}{\Gamma(\delta)} \int_{0}^{\ell}(\ell-\mathrm{s})^{\delta-1} \overline{\mathcal{F}}\left(\mathrm{s}, \mathrm{u}(\mathrm{s}), D^{\delta} \mathrm{w}(\mathrm{s})\right) d \mathrm{~s} .
\end{aligned}\right.
$$

Let $\mathcal{A}=\left(\mathcal{A}_{1}, \mathcal{A}_{2}\right), \mathcal{B}=\left(\mathcal{B}_{1}, \mathcal{B}_{2}\right)$, and $\mathcal{T}=\mathcal{A}+\mathcal{B}$, where $\mathcal{A}_{1}: \mathcal{M} \rightarrow \mathcal{M}$ and $\mathcal{A}_{2}: \mathcal{N} \rightarrow \mathcal{N}$ are defined by

$$
\mathcal{A}_{1}(\mathrm{u})(\ell)=\left(1-\ell^{2}\right) r(\mathrm{u})+\left(\ell-\ell^{2}\right) \mathrm{u}_{o}
$$


and

$$
\mathcal{A}_{2}(\mathrm{w})(\ell)=\left(1-\ell^{2}\right) h(\mathrm{w})+\left(\ell-\ell^{2}\right) \mathrm{w}_{o}
$$

and $\mathcal{B}_{1}, \mathcal{B}_{2}: \mathcal{M} \times \mathcal{N} \rightarrow \mathcal{M} \times \mathcal{N}$ are defined by

$$
\begin{aligned}
\mathcal{B}_{1}(\mathrm{u}, \mathrm{w})(\ell)= & \frac{\ell^{2}}{\Gamma(\kappa)} \int_{0}^{1}(1-\mathrm{s})^{\kappa-1} \mathrm{~g}_{1}(\mathrm{~s}, \mathrm{u}(\mathrm{s})) d \mathrm{~s} \\
& -\frac{\ell^{2}}{\Gamma(\kappa)} \int_{0}^{1}(1-\mathrm{s})^{\kappa-1} \mathcal{F}\left(\mathrm{s}, \mathrm{w}(\mathrm{s}), D^{\kappa} \mathrm{u}(\mathrm{s})\right) d \mathrm{~s} \\
& +\frac{1}{\Gamma(\kappa)} \int_{0}^{\ell}(\ell-\mathrm{s})^{\kappa-1} \mathcal{F}\left(\mathrm{s}, \mathrm{w}(\mathrm{s}), D^{\kappa} \mathrm{u}(\mathrm{s})\right) d \mathrm{~s}
\end{aligned}
$$

and

$$
\begin{aligned}
\mathcal{B}_{2}(\mathrm{u}, \mathrm{w})(\ell)= & \frac{\ell^{2}}{\Gamma(\delta)} \int_{0}^{1}(1-\mathrm{s})^{\delta-1} \mathrm{~g}_{2}(\mathrm{~s}, \mathrm{w}(\mathrm{s})) d \mathrm{~s}-\frac{\ell^{2}}{\Gamma(\delta)} \int_{0}^{1}(1-\mathrm{s})^{\delta-1} \overline{\mathcal{F}}\left(\mathrm{s}, \mathrm{u}(\mathrm{s}), D^{\delta} \mathrm{w}(\mathrm{s})\right) d \mathrm{~s} \\
& +\frac{1}{\Gamma(\delta)} \int_{0}^{\ell}(\ell-\mathrm{s})^{\delta-1} \overline{\mathcal{F}}\left(\mathrm{s}, \mathrm{u}(\mathrm{s}), D^{\delta} \mathrm{w}(\mathrm{s})\right) d \mathrm{~s} .
\end{aligned}
$$

Then the solution of (1.1) in the operator form becomes

$$
(\mathrm{u}, \mathrm{w})=\mathcal{T}(\mathrm{u}, \mathrm{w})=\mathcal{A}(\mathrm{u}, \mathrm{w})+\mathcal{B}(\mathrm{u}, \mathrm{w}) .
$$

Lemma 3.2 The following Lipschitz condition is satisfied for the operator $\mathcal{A}$ :

$$
|\mathcal{A}(\mathrm{u}, \mathrm{w})(\ell)-\mathcal{A}(\overline{\mathrm{u}}, \overline{\mathrm{w}})(\ell)| \leq k_{\theta}\|(\mathrm{u}, \mathrm{w})-(\overline{\mathrm{u}}, \overline{\mathrm{w}})\|
$$

Proof For any $(\mathrm{u}, \mathrm{w}),(\overline{\mathrm{u}}, \overline{\mathrm{w}}) \in \mathcal{M} \times \mathcal{N}$, we have

$$
\begin{aligned}
|\mathcal{A}(\mathrm{u}, \mathrm{w})(\ell)-\mathcal{A}(\overline{\mathrm{u}}, \overline{\mathrm{w}})(\ell)| & =\left|\left(1-\ell^{2}\right) r(\mathrm{u})+\left(1-\ell^{2}\right) h(\mathrm{w})-\left(1-\ell^{2}\right) r(\overline{\mathrm{u}})-\left(1-\ell^{2}\right) h(\overline{\mathrm{w}})\right| \\
& \leq \mid\left(1-\ell^{2}\right)\left(r(\mathrm{u})-r(\overline{\mathrm{u}})|+|\left(1-\ell^{2}\right)(h(\mathrm{w})-h(\overline{\mathrm{w}}) \mid\right. \\
& \leq|r(\mathrm{u})-r(\overline{\mathrm{u}})|+|h(\mathrm{w})-h(\overline{\mathrm{w}})| \\
& \leq k_{r}|\mathrm{u}-\overline{\mathrm{u}}|+k_{h}|\mathrm{w}-\overline{\mathrm{w}}|
\end{aligned}
$$

which implies that

$$
|\mathcal{A}(\mathrm{u}, \mathrm{w})(\ell)-\mathcal{A}(\overline{\mathrm{u}}, \overline{\mathrm{w}})(\ell)| \leq k_{\theta}\|(\mathrm{u}, \mathrm{w})-(\overline{\mathrm{u}}, \overline{\mathrm{w}})\|
$$

where $k_{\theta}=\max \left\{k_{r}, k_{h}\right\}$. Thus $\mathcal{A}$ is Lipschitz having constant $k_{\theta}$, and in view of Proposition 2.2, $\mathcal{A}$ is $\sigma$-Lipschitz having constant $k_{\theta}$.

Lemma 3.3 The operator $\mathcal{B}: \mathcal{M} \times \mathcal{N} \rightarrow \mathcal{M} \times \mathcal{N}$ is continuous.

Proof Let $\left\{\left(\mathrm{u}_{n}, \mathrm{w}_{n}\right)\right\}$ be a sequence in a bounded set

$$
D_{k}=\{\|(\mathrm{u}, \mathrm{w})\| \leq r:(\mathrm{u}, \mathrm{w}) \in \mathcal{M} \times \mathcal{N}\},
$$


so that $\left(\mathrm{u}_{n}, \mathrm{w}_{n}\right) \rightarrow(\mathrm{u}, \mathrm{w})$ as $n \rightarrow \infty$ in $D_{k}$. To check the continuity of $\mathcal{B}$, we have to show that

$$
\left\|\mathcal{B}\left(\mathrm{u}_{n}, \mathrm{w}_{n}\right)-\mathcal{B}(\mathrm{u}, \mathrm{w})\right\| \rightarrow 0 \quad \text { as } n \rightarrow \infty .
$$

For this, we have

$$
\begin{aligned}
\left|\mathcal{B}_{1}\left(\mathrm{u}_{n}, \mathrm{w}_{n}\right)(\ell)-\mathcal{B}_{1}(\mathrm{u}, \mathrm{w})(\ell)\right| & \\
= & \frac{\ell^{2}}{\Gamma(\kappa)} \int_{0}^{1}(1-\mathrm{s})^{\kappa-1} \mathrm{~g}_{1}\left(\mathrm{~s}, \mathrm{u}_{n}(\mathrm{~s})\right) d \mathrm{~s}-\frac{\ell^{2}}{\Gamma(\kappa)} \int_{0}^{1}(1-\mathrm{s})^{\kappa-1} \mathcal{F}\left(\mathrm{s}, \mathrm{w}_{n}(\mathrm{~s}), D^{\kappa} \mathrm{u}_{n}(\mathrm{~s})\right) d \mathrm{~s} \\
& +\frac{1}{\Gamma(\kappa)} \int_{0}^{\ell}(\ell-\mathrm{s})^{\kappa-1} \mathcal{F}\left(\mathrm{s}, \mathrm{w}_{n}(\mathrm{~s}), D^{\kappa} \mathrm{u}_{n}(\mathrm{~s})\right) d \mathrm{~s}-\frac{\ell^{2}}{\Gamma(\kappa)} \int_{0}^{1}(1-\mathrm{s})^{\kappa-1} \mathrm{~g}_{1}(\mathrm{~s}, \mathrm{u}(\mathrm{s})) d \mathrm{~s} \\
& +\frac{\ell^{2}}{\Gamma(\kappa)} \int_{0}^{1}(1-\mathrm{s})^{\kappa-1} \mathcal{F}\left(\mathrm{s}, \mathrm{w}(\mathrm{s}), D^{\kappa} \mathrm{u}(\mathrm{s})\right) d \mathrm{~s} \\
& -\frac{1}{\Gamma(\kappa)} \int_{0}^{\ell}(\ell-\mathrm{s})^{\kappa-1} \mathcal{F}\left(\mathrm{s}, \mathrm{w}(\mathrm{s}), D^{\kappa} \mathrm{u}(\mathrm{s})\right) d \mathrm{~s} \\
\leq & \frac{1}{\Gamma(\kappa)} \int_{0}^{1}(1-\mathrm{s})^{\kappa-1} \mathrm{~g}_{1}\left(\mathrm{~s}, \mathrm{u}_{n}(\mathrm{~s})\right) d \mathrm{~s}-\frac{1}{\Gamma(\kappa)} \int_{0}^{1}(1-\mathrm{s})^{\kappa-1} \mathrm{~g}_{1}(\mathrm{~s}, \mathrm{u}(\mathrm{s})) d \mathrm{~s} \\
& -\frac{1}{\Gamma(\kappa)} \int_{0}^{1}(1-\mathrm{s})^{\kappa-1} \mathcal{F}\left(\mathrm{s}, \mathrm{w}_{n}(\mathrm{~s}), D^{\kappa} \mathrm{u}_{n}(\mathrm{~s})\right) d \mathrm{~s} \\
& +\frac{1}{\Gamma(\kappa)} \int_{0}^{1}(1-\mathrm{s})^{\kappa-1} \mathcal{F}\left(\mathrm{s}, \mathrm{w}(\mathrm{s}), D^{\kappa} \mathrm{u}(\mathrm{s})\right) d \mathrm{~s} \\
& +\frac{1}{\Gamma(\kappa)} \int_{0}^{\ell}(\ell-\mathrm{s})^{\kappa-1} \mathcal{F}\left(\mathrm{s}, \mathrm{w}_{n}(\mathrm{~s}), D^{\kappa} \mathrm{u}_{n}(\mathrm{~s})\right) d \mathrm{~s} \\
& -\frac{1}{\Gamma(\kappa)} \int_{0}^{\ell}(\ell-\mathrm{s})^{\kappa-1} \mathcal{F}\left(\mathrm{s}, \mathrm{w}(\mathrm{s}), D^{\kappa} \mathrm{u}(\mathrm{s})\right) d \mathrm{~s} \mid
\end{aligned}
$$

which implies that

$$
\begin{aligned}
\left|\mathcal{B}_{1}\left(\mathrm{u}_{n}, \mathrm{w}_{n}\right)(\ell)-\mathcal{B}_{1}(\mathrm{u}, \mathrm{w})(\ell)\right| & \\
\leq & \left|\frac{1}{\Gamma(\kappa)} \int_{0}^{1}(1-\mathrm{s})^{\kappa-1} \mathrm{~g}_{1}\left(\mathrm{~s}, \mathrm{u}_{n}(\mathrm{~s})\right) d \mathrm{~s}-\frac{1}{\Gamma(\kappa)} \int_{0}^{1}(1-\mathrm{s})^{\kappa-1} \mathrm{~g}_{1}(\mathrm{~s}, \mathrm{u}(\mathrm{s})) d \mathrm{~s}\right| \\
& +\mid \frac{1}{\Gamma(\kappa)} \int_{0}^{1}(1-\mathrm{s})^{\kappa-1} \mathcal{F}\left(\mathrm{s}, \mathrm{w}_{n}(\mathrm{~s}), D^{\kappa} \mathrm{u}_{n}(\mathrm{~s})\right) d \mathrm{~s} \\
& -\frac{1}{\Gamma(\kappa)} \int_{0}^{1}(1-\mathrm{s})^{\kappa-1} \mathcal{F}\left(\mathrm{s}, \mathrm{w}(\mathrm{s}), D^{\kappa} \mathrm{u}(\mathrm{s})\right) d \mathrm{~s} \mid \\
& +\mid \frac{1}{\Gamma(\kappa)} \int_{0}^{\ell}(\ell-\mathrm{s})^{\kappa-1} \mathcal{F}\left(\mathrm{s}, \mathrm{w}_{n}(\mathrm{~s}), D^{\kappa} \mathrm{u}_{n}(\mathrm{~s})\right) d \mathrm{~s} \\
& -\frac{1}{\Gamma(\kappa)} \int_{0}^{\ell}(\ell-\mathrm{s})^{\kappa-1} \mathcal{F}\left(\mathrm{s}, \mathrm{w}(\mathrm{s}), D^{\kappa} \mathrm{u}(\mathrm{s})\right) d \mathrm{~s} \mid \\
= & \frac{1}{\Gamma(\kappa)} \int_{0}^{1}(1-\mathrm{s})^{\kappa-1}\left|\mathrm{~g}_{1}\left(\mathrm{~s}, \mathrm{u}_{n}(\mathrm{~s})\right)-\mathrm{g}_{1}(\mathrm{~s}, \mathrm{u}(\mathrm{s}))\right| d \mathrm{~s}
\end{aligned}
$$




$$
\begin{aligned}
& +\frac{1}{\Gamma(\kappa)} \int_{0}^{1}(1-\mathrm{s})^{\kappa-1}\left|\mathcal{F}\left(\mathrm{s}, \mathrm{w}_{n}(\mathrm{~s}), D^{\kappa} \mathrm{u}_{n}(\mathrm{~s})\right)-\mathcal{F}\left(\mathrm{s}, \mathrm{w}(\mathrm{s}), D^{\kappa} \mathrm{u}(\mathrm{s})\right)\right| d \mathrm{~s} \\
& +\frac{1}{\Gamma(\kappa)} \int_{0}^{\ell}(\ell-\mathrm{s})^{\kappa-1}\left|\mathcal{F}\left(\mathrm{s}, \mathrm{w}_{n}(\mathrm{~s}), D^{\kappa} \mathrm{u}_{n}(\mathrm{~s})\right)-\mathcal{F}\left(\mathrm{s}, \mathrm{w}(\mathrm{s}), D^{\kappa} \mathrm{u}(\mathrm{s})\right)\right| d \mathrm{~s}
\end{aligned}
$$

From the continuity of $\mathcal{F}$ it follows that

$$
\mathcal{F}\left(\mathrm{s}, \mathrm{w}_{n}(\mathrm{~s}), \omega_{n}(\mathrm{~s})\right) \rightarrow \mathcal{F}(\mathrm{s}, \mathrm{w}(\mathrm{s}), \omega(\mathrm{s})) \quad \text { as } n \rightarrow \infty
$$

For each $\ell \in \mathfrak{J}$, using $\left(C_{5}\right)$ we obtain

$$
\int_{0}^{\ell} \frac{(\ell-\mathrm{s})^{\kappa-1}}{\Gamma(\kappa)}\left|\mathcal{F}\left(\mathrm{s}, \mathrm{w}_{n}(\mathrm{~s}), \omega_{n}(\mathrm{~s})\right)-\mathcal{F}(\mathrm{s}, \mathrm{w}(\mathrm{s}), \omega(\mathrm{s}))\right| d \mathrm{~s} \rightarrow 0 \quad \text { as } n \rightarrow \infty
$$

similarly other terms approach 0 as $n \rightarrow \infty$. It follows that

$$
\left|\mathcal{B}_{1}\left(\mathrm{u}_{n}, \mathrm{w}_{n}\right)(\ell)-\mathcal{B}_{1}(\mathrm{u}, \mathrm{w})(\ell)\right| \rightarrow 0 \quad \text { as } n \rightarrow \infty
$$

Similarly,

$$
\left|\mathcal{B}_{2}\left(\mathrm{u}_{n}, \mathrm{w}_{n}\right)(\ell)-\mathcal{B}_{2}(\mathrm{u}, \mathrm{w})(\ell)\right| \rightarrow 0 \quad \text { as } n \rightarrow \infty
$$

Therefore $\mathcal{B}_{1}$ and $\mathcal{B}_{2}$ and thus $\mathcal{B}$ is continuous.

Lemma 3.4 The following growth conditions are valid for the operators $\mathcal{A}$ and $\mathcal{B}$ :

$$
\|\mathcal{A}(\mathrm{u}, \mathrm{w})\| \leq c_{\theta}\|(\mathrm{u}, \mathrm{w})\|+M \quad \text { for each }(\mathrm{u}, \mathrm{w}) \in \mathcal{M} \times \mathcal{N}
$$

and

$$
\|\mathcal{B}(\mathrm{u}, \mathrm{w})\| \leq \theta\|(\mathrm{u}, \mathrm{w})\|+\Lambda \quad \text { for each }(\mathrm{u}, \mathrm{w}) \in \mathcal{M} \times \mathcal{N}
$$

respectively, where $c_{\theta}=\max \left\{c_{r}, c_{h}\right\}, \theta=\max \left\{z_{\mathrm{g}_{1}}+\frac{2 d_{1}}{1-d_{2}}, z_{\mathrm{g}_{2}}+\frac{2 c_{1}}{1-c_{2}}\right\}$, and $\Lambda=\frac{2 M_{\mathcal{F}}}{1-c_{2}}+\frac{2 M_{\overline{\mathcal{F}}}}{1-d_{2}}+$ $N_{\mathrm{g}_{1}}+N_{\mathrm{g}_{2}}$.

Proof For the growth condition, consider

$$
\begin{aligned}
|\mathcal{A}(\mathrm{u}, \mathrm{w})| & =\left|\left(\mathcal{A}_{1}(\mathrm{u}), \mathcal{A}_{2}(\mathrm{w})\right)\right| \\
& =\left|\left(1-\ell^{2}\right) r(\mathrm{u})+\left(\ell-\ell^{2}\right) \mathrm{u}_{o}+\left(1-\ell^{2}\right) h(\mathrm{w})+\left(\ell-\ell^{2}\right) \mathrm{w}_{o}\right| \\
& \leq\left|r(\mathrm{u})+\mathrm{u}_{o}\right|+\left|h(\mathrm{w})+\mathrm{w}_{o}\right| \\
& \leq|r(\mathrm{u})|+|h(\mathrm{w})|+\left|\mathrm{u}_{o}\right|+\left|\mathrm{w}_{o}\right| \\
& \leq c_{r}|\mathrm{u}|+c_{h}|\mathrm{w}|+M_{r}+M_{h}+\left|\mathrm{u}_{o}\right|+\left|\mathrm{w}_{o}\right| \\
& \leq c_{\theta}\|(\mathrm{u}, \mathrm{w})\|+M,
\end{aligned}
$$


where $M=M_{r}+M_{h}+\left|\mathrm{u}_{o}\right|+\left|\mathrm{w}_{o}\right|$, hence the operator $\mathcal{A}$ satisfies the growth condition. Now

$$
\begin{aligned}
&\left\|\mathcal{B}_{1}(\mathrm{u}, \mathrm{w})(\ell)\right\| \\
&=\| \frac{\ell^{2}}{\Gamma(\kappa)} \int_{0}^{1}(1-\mathrm{s})^{\kappa-1} \mathrm{~g}_{1}(\mathrm{~s}, \mathrm{u}(\mathrm{s})) d \mathrm{~s}-\frac{\ell^{2}}{\Gamma(\kappa)} \int_{0}^{1}(1-\mathrm{s})^{\kappa-1} \mathcal{F}\left(\mathrm{s}, \mathrm{w}(\mathrm{s}), D^{\kappa} \mathrm{u}(\mathrm{s})\right) d \mathrm{~s} \\
& \quad+\frac{1}{\Gamma(\kappa)} \int_{0}^{\ell}(\ell-\mathrm{s})^{\kappa-1} \mathcal{F}\left(\mathrm{s}, \mathrm{w}(\mathrm{s}), D^{\kappa} \mathrm{u}(\mathrm{s})\right) d \mathrm{~s} \| \\
& \leq \frac{1}{\Gamma(\kappa)} \int_{0}^{1}(1-\mathrm{s})^{\kappa-1}\left\|\mathrm{~g}_{1}(\mathrm{~s}, \mathrm{u}(\mathrm{s}))\right\| d \mathrm{~s}+\frac{1}{\Gamma(\kappa)} \int_{0}^{1}(1-\mathrm{s})^{\kappa-1}\left\|\mathcal{F}\left(\mathrm{s}, \mathrm{w}(\mathrm{s}), D^{\kappa} \mathrm{u}(\mathrm{s})\right)\right\| d \mathrm{~s} \\
& \quad+\frac{1}{\Gamma(\kappa)} \int_{0}^{\ell}(\ell-\mathrm{s})^{\kappa-1}\left\|\mathcal{F}\left(\mathrm{s}, \mathrm{w}(\mathrm{s}), D^{\kappa} \mathrm{u}(\mathrm{s})\right)\right\| d \mathrm{~s} \\
& \leq z_{\mathrm{g}_{1}}|\mathrm{u}|+2 c_{1}|\mathrm{w}|+2 c_{2}|\omega|+N_{\mathrm{g}_{1}}+2 M_{\mathcal{F}} \\
& \leq z_{\mathrm{g}_{1}}|\mathrm{u}|+\frac{2 c_{1}}{1-c_{2}}|\mathrm{w}|+\frac{2 M_{\mathcal{F}}}{1-c_{2}}+N_{\mathrm{g}_{1}},
\end{aligned}
$$

similarly

$$
\left\|\mathcal{B}_{2}(\mathrm{u}, \mathrm{w})(\ell)\right\| \leq z_{\mathrm{g}_{2}}|\mathrm{w}|+\frac{2 d_{1}}{1-d_{2}}|\mathrm{u}|+\frac{2 M_{\overline{\mathcal{F}}}}{1-d_{2}}+N_{\mathrm{g}_{2}} .
$$

Now

$$
\begin{aligned}
\|\mathcal{B}(\mathrm{u}, \mathrm{w})\| & =\left\|\mathcal{B}_{1}(\mathrm{u}, \mathrm{w})\right\|+\left\|\mathcal{B}_{2}(\mathrm{u}, \mathrm{w})\right\| \\
& \leq z_{\mathrm{g}_{1}}|\mathrm{u}|+z_{\mathrm{g}_{2}}|\mathrm{w}|+\frac{2 d_{1}}{1-d_{2}}|\mathrm{u}|+\frac{2 c_{1}}{1-c_{2}}|\mathrm{w}|+\frac{2 M_{\mathcal{F}}}{1-c_{2}}+\frac{2 M_{\overline{\mathcal{F}}}}{1-d_{2}}+N_{\mathrm{g}_{1}}+N_{\mathrm{g}_{2}} \\
& \leq\left(z_{\mathrm{g}_{1}}+\frac{2 d_{1}}{1-d_{2}}\right)|\mathrm{u}|+\left(z_{\mathrm{g}_{2}}+\frac{2 c_{1}}{1-c_{2}}\right)|\mathrm{w}|+\frac{2 M_{\mathcal{F}}}{1-c_{2}}+\frac{2 M_{\overline{\mathcal{F}}}}{1-d_{2}}+N_{\mathrm{g}_{1}}+N_{\mathrm{g}_{2}}
\end{aligned}
$$

which implies that

$$
\|\mathcal{B}(\mathrm{u}, \mathrm{w})\| \leq \theta\|(\mathrm{u}, \mathrm{w})\|+\Lambda
$$

which is the required growth condition on $\mathcal{B}$.

Lemma 3.5 The operator $\mathcal{B}: \mathcal{M} \times \mathcal{N} \rightarrow \mathcal{M} \times \mathcal{N}$ is compact. Consequently, $\mathcal{B}$ is $\sigma$ Lipschitz with the constant zero.

Proof Consider a sequence $\left\{\left(\mathrm{u}_{n}, \mathrm{w}_{n}\right)\right\}_{n \in \mathbb{N}}$ in $\mathfrak{D}$, where $\mathfrak{D}$ is a bounded subset of $D_{k}$. Then, by using the growth condition of $\mathcal{B}(3.7)$, it is clear that $G(\mathfrak{D})$ is bounded. Now, we will show that $\mathcal{B}$ is equicontinuous. For each $\left\{\left(\mathrm{u}_{n}, \mathrm{w}_{n}\right)\right\}$ in $\mathfrak{D}$ and for each $\epsilon>0$, we have

$$
\begin{aligned}
& \left|\mathcal{B}_{1}\left(\mathrm{u}_{n}, \mathrm{w}_{n}\right)(\ell)-\mathcal{B}_{1}\left(\mathrm{u}_{n}, \mathrm{w}_{n}\right)(\tau)\right| \\
& =\mid \frac{\ell^{2}}{\Gamma(\kappa)} \int_{0}^{1}(1-\mathrm{s})^{\kappa-1} \mathrm{~g}_{1}\left(\mathrm{~s}, \mathrm{u}_{n}(\mathrm{~s})\right) d \mathrm{~s}-\frac{\ell^{2}}{\Gamma(\kappa)} \int_{0}^{1}(1-\mathrm{s})^{\kappa-1} \mathcal{F}\left(\mathrm{s}, \mathrm{w}_{n}(\mathrm{~s}), D^{\kappa} \mathrm{u}_{n}(\mathrm{~s})\right) d \mathrm{~s} \\
& \quad+\frac{1}{\Gamma(\kappa)} \int_{0}^{\ell}(\ell-\mathrm{s})^{\kappa-1} \mathcal{F}\left(\mathrm{s}, \mathrm{w}_{n}(\mathrm{~s}), D^{\kappa} \mathrm{u}_{n}(\mathrm{~s})\right) d \mathrm{~s}-\frac{\tau^{2}}{\Gamma(\kappa)} \int_{0}^{1}(1-\mathrm{s})^{\kappa-1} \mathrm{~g}_{1}\left(\mathrm{~s}, \mathrm{u}_{n}(\mathrm{~s})\right) d \mathrm{~s}
\end{aligned}
$$




$$
\begin{aligned}
& +\frac{\tau^{2}}{\Gamma(\kappa)} \int_{0}^{1}(1-\mathrm{s})^{\kappa-1} \mathcal{F}\left(\mathrm{s}, \mathrm{w}_{n}(\mathrm{~s}), D^{\kappa} \mathrm{u}_{n}(\mathrm{~s})\right) d \mathrm{~s} \\
& -\frac{1}{\Gamma(\kappa)} \int_{0}^{\ell}(\tau-\mathrm{s})^{\kappa-1} \mathcal{F}\left(\mathrm{s}, \mathrm{w}_{n}(\mathrm{~s}), D^{\kappa} \mathrm{u}_{n}(\mathrm{~s})\right) d \mathrm{~s} \\
& -\frac{1}{\Gamma(\kappa)} \int_{\ell}^{\tau}(\tau-\mathrm{s})^{\kappa-1} \mathcal{F}\left(\mathrm{s}, \mathrm{w}_{n}(\mathrm{~s}), D^{\kappa} \mathrm{u}_{n}(\mathrm{~s})\right) d \mathrm{~s} \mid
\end{aligned}
$$

which implies that

$$
\begin{aligned}
& \left|\mathcal{B}_{1}\left(\mathrm{u}_{n}, \mathrm{w}_{n}\right)(\ell)-\mathcal{B}_{1}\left(\mathrm{u}_{n}, \mathrm{w}_{n}\right)(\tau)\right| \\
& \leq\left|\frac{\ell^{2}}{\Gamma(\kappa)} \int_{0}^{1}(1-\mathrm{s})^{\kappa-1} \mathrm{~g}_{1}\left(\mathrm{~s}, \mathrm{u}_{n}(\mathrm{~s})\right) d \mathrm{~s}-\frac{\tau^{2}}{\Gamma(\kappa)} \int_{0}^{1}(1-\mathrm{s})^{\kappa-1} \mathrm{~g}_{1}\left(\mathrm{~s}, \mathrm{u}_{n}(\mathrm{~s})\right) d \mathrm{~s}\right| \\
& +\mid \frac{1}{\Gamma(\kappa)} \int_{0}^{\ell}(\ell-\mathrm{s})^{\kappa-1} \mathcal{F}\left(\mathrm{s}, \mathrm{w}_{n}(\mathrm{~s}), D^{\kappa} \mathrm{u}_{n}(\mathrm{~s})\right) d \mathrm{~s} \\
& -\frac{1}{\Gamma(\kappa)} \int_{0}^{\ell}(\tau-\mathrm{s})^{\kappa-1} \mathcal{F}\left(\mathrm{s}, \mathrm{w}_{n}(\mathrm{~s}), D^{\kappa} \mathrm{u}_{n}(\mathrm{~s})\right) d \mathrm{~s} \\
& +\mid \frac{\tau^{2}}{\Gamma(\kappa)} \int_{0}^{1}(1-\mathrm{s})^{\kappa-1} \mathcal{F}\left(\mathrm{s}, \mathrm{w}_{n}(\mathrm{~s}), D^{\kappa} \mathrm{u}_{n}(\mathrm{~s})\right) d \mathrm{~s} \\
& -\frac{\ell^{2}}{\Gamma(\kappa)} \int_{0}^{1}(1-\mathrm{s})^{\kappa-1} \mathcal{F}\left(\mathrm{s}, \mathrm{w}_{n}(\mathrm{~s}), D^{\kappa} \mathrm{u}_{n}(\mathrm{~s})\right) d \mathrm{~s} \\
& +\left|\frac{1}{\Gamma(\kappa)} \int_{\ell}^{\tau}(\tau-\mathrm{s})^{\kappa-1} \mathcal{F}\left(\mathrm{s}, \mathrm{w}_{n}(\mathrm{~s}), D^{\kappa} \mathrm{u}_{n}(\mathrm{~s})\right) d \mathrm{~s}\right| \\
& =\left|\frac{1}{\Gamma(\kappa)} \int_{0}^{\ell}\left((\ell-\mathrm{s})^{\kappa-1}-(\tau-\mathrm{s})^{\kappa-1}\right) \mathcal{F}\left(\mathrm{s}, \mathrm{w}_{n}(\mathrm{~s}), D^{\kappa} \mathrm{u}_{n}(\mathrm{~s})\right) d \mathrm{~s}\right| \\
& +\left|\frac{\left(\tau^{2}-\ell^{2}\right)}{\Gamma(\kappa)} \int_{0}^{1}(1-\mathrm{s})^{\kappa-1} \mathcal{F}\left(\mathrm{s}, \mathrm{w}_{n}(\mathrm{~s}), D^{\kappa} \mathrm{u}_{n}(\mathrm{~s})\right) d \mathrm{~s}\right| \\
& +\left|\frac{1}{\Gamma(\kappa)} \int_{\ell}^{\tau}(\tau-\mathrm{s})^{\kappa-1} \mathcal{F}\left(\mathrm{s}, \mathrm{w}_{n}(\mathrm{~s}), D^{\kappa} \mathrm{u}_{n}(\mathrm{~s})\right) d \mathrm{~s}\right| \\
& +\left|\frac{\left(\ell^{2}-\tau^{2}\right)}{\Gamma(\kappa)} \int_{0}^{1}(1-\mathrm{s})^{\kappa-1} \mathrm{~g}_{1}\left(\mathrm{~s}, \mathrm{u}_{n}(\mathrm{~s})\right) d \mathrm{~s}\right| \\
& =\frac{\left|\left(\ell^{2}-\tau^{2}\right)\right|\left\|g_{1}\right\|}{\Gamma(\kappa)} \int_{0}^{1}(1-\mathrm{s})^{\kappa-1} d \mathrm{~s}+\frac{\|\mathcal{F}\|}{\Gamma(\kappa)} \int_{0}^{\ell}\left|(\ell-\mathrm{s})^{\kappa-1}-(\tau-\mathrm{s})^{\kappa-1}\right| d \mathrm{~s} \\
& +\frac{\left(\tau^{2}-\ell^{2}\right)\|\mathcal{F}\|}{\Gamma(\kappa)} \int_{0}^{1}(1-\mathrm{s})^{\kappa-1} d \mathrm{~s}+\frac{\|\mathcal{F}\|}{\Gamma(\kappa)} \int_{\ell}^{\tau}(\tau-\mathrm{s})^{\kappa-1} d \mathrm{~s} \\
& =\frac{2\|\mathcal{F}\|}{\Gamma(\kappa+1)}(\tau-\ell)^{\kappa}-\frac{\left\|g_{1}\right\|}{\Gamma(\kappa+1)}\left(\tau^{2}-\ell^{2}\right) \text {. }
\end{aligned}
$$

Taking limit as $\tau \rightarrow \ell$, we get

$$
\left|\mathcal{B}_{1}\left(\mathrm{u}_{n}, \mathrm{w}_{n}\right)(\ell)-\mathcal{B}_{1}\left(\mathrm{u}_{n}, \mathrm{w}_{n}\right)(\tau)\right| \rightarrow 0 .
$$

So there exists $\epsilon>0$ such that

$$
\left|\mathcal{B}_{1}\left(\mathrm{u}_{n}, \mathrm{w}_{n}\right)(\ell)-\mathcal{B}_{1}\left(\mathrm{u}_{n}, \mathrm{w}_{n}\right)(\tau)\right|<\frac{\epsilon}{2},
$$


similarly

$$
\left|\mathcal{B}_{2}\left(\mathrm{u}_{n}, \mathrm{w}_{n}\right)(\ell)-\mathcal{B}_{2}\left(\mathrm{u}_{n}, \mathrm{w}_{n}\right)(\tau)\right|<\frac{\epsilon}{2} .
$$

Therefore, from (3.10) and (3.11), we get

$$
\left|\mathcal{B}\left(\mathrm{u}_{n}, \mathrm{w}_{n}\right)(\ell)-\mathcal{B}\left(\mathrm{u}_{n}, \mathrm{w}_{n}\right)(\tau)\right|<\epsilon .
$$

Thus $\mathcal{B}$ is equicontinuous, and therefore $\mathcal{B}(\mathfrak{D})$ is compact in $\mathcal{M} \times \mathcal{N}$. In view of Proposition 2.3, $\mathcal{B}$ is $\sigma$-Lipschitz having zero constant.

Theorem 3.1 Under assumptions $\left(C_{1}\right)-\left(C_{4}\right), B V P(1.1)$ has at least one solution $(\mathrm{u}, \mathrm{w}) \in$ $\mathcal{M} \times \mathcal{N}$ provided $c_{\theta}+\theta<1$ and a solution set of $(1.1)$ is bounded in $\mathcal{M} \times \mathcal{N}$.

Proof By Lemma 3.2, $\mathcal{A}$ is Lipschitz having constant $k_{\theta} \in[0,1)$, and by Lemma $3.5, \mathcal{B}$ is Lipschitz having zero constant. Therefore, by Proposition 2.1, $\mathcal{T}$ is a $\sigma$-contraction having constant $k_{\theta}$. Now define

$$
\mathcal{Q}=\{(\mathrm{u}, \mathrm{w}) \in \mathcal{M} \times \mathcal{N}: \exists \varrho \in \mathfrak{J}, \ni(\mathrm{u}, \mathrm{w})=\varrho \mathcal{T}(\mathrm{u}, \mathrm{w})\}
$$

We have to prove that $\mathcal{Q}$ is bounded in $\mathcal{M} \times \mathcal{N}$. So, choose $(\mathrm{u}, \mathrm{w}) \in \mathcal{Q}$, then by using (3.6) and (3.7), we have

$$
\begin{aligned}
\|(\mathrm{u}, \mathrm{w})\| & =\|\varrho \mathcal{T}(\mathrm{u}, \mathrm{w})\| \\
& =\varrho(\|\mathcal{A}(\mathrm{u}, \mathrm{w})+\mathcal{B}(\mathrm{u}, \mathrm{w})\|) \\
& \leq \varrho\left(c_{\theta}\|(\mathrm{u}, \mathrm{w})\|+M+\theta(\|(\mathrm{u}, \mathrm{w})\|+\Lambda)\right. \\
& =\varrho\left(c_{\theta}+\theta\right)\|(\mathrm{u}, \mathrm{w})\|+\varrho(M+\Lambda) .
\end{aligned}
$$

Thus $\mathcal{Q}$ is bounded in $\mathcal{M} \times \mathcal{N}$. Therefore Theorem 2.1 guarantees that $\mathcal{T}$ possesses at least one fixed point. Hence the considered problem has at least one solution.

Theorem 3.2 Suppose that $\left(k_{\theta}+C^{\prime}+D^{\prime}\right)<1$. Let assumptions $\left(C_{1}\right),\left(C_{5}\right)$, and $\left(C_{6}\right)$ be satisfied. Then BVP (1.1) has a unique solution.

Proof In the light of Banach contraction theorem, for any $(\mathrm{u}, \mathrm{w}),(\overline{\mathrm{u}}, \overline{\mathrm{w}}) \in \mathcal{M} \times \mathcal{N}$, consider

$$
\begin{aligned}
& \left|\mathcal{B}_{1}(\mathrm{u}, \mathrm{w})-\mathcal{B}_{1}(\overline{\mathrm{u}}, \overline{\mathrm{w}})\right| \\
& =\mid \frac{\ell^{2}}{\Gamma(\kappa)} \int_{0}^{1}(1-\mathrm{s})^{\kappa-1} \mathrm{~g}_{1}(\mathrm{~s}, \mathrm{u}(\mathrm{s})) d \mathrm{~s}+\frac{1}{\Gamma(\kappa)} \int_{0}^{\ell}(\ell-\mathrm{s})^{\kappa-1} \mathcal{F}\left(\mathrm{s}, \mathrm{w}(\mathrm{s}), D^{\kappa} \mathrm{u}(\mathrm{s})\right) d \mathrm{~s} \\
& \quad-\frac{\ell^{2}}{\Gamma(\kappa)} \int_{0}^{1}(1-\mathrm{s})^{\kappa-1} \mathcal{F}\left(\mathrm{s}, \mathrm{w}(\mathrm{s}), D^{\kappa} \mathrm{u}(\mathrm{s})\right) d \mathrm{~s}-\frac{\ell^{2}}{\Gamma(\kappa)} \int_{0}^{1}(1-\mathrm{s})^{\kappa-1} \mathrm{~g}_{1}(\mathrm{~s}, \overline{\mathrm{u}}(\mathrm{s})) d \mathrm{~s} \\
& \quad-\frac{1}{\Gamma(\kappa)} \int_{0}^{\ell}(\ell-\mathrm{s})^{\kappa-1} \mathcal{F}\left(\mathrm{s}, \overline{\mathrm{w}}(\mathrm{s}), D^{\kappa} \overline{\mathrm{u}}(\mathrm{s})\right) d \mathrm{~s} \\
& \quad+\frac{\ell^{2}}{\Gamma(\kappa)} \int_{0}^{1}(1-\mathrm{s})^{\kappa-1} \mathcal{F}\left(\mathrm{s}, \overline{\mathrm{w}}(\mathrm{s}), D^{\kappa} \overline{\mathrm{u}}(\mathrm{s})\right) d \mathrm{~s} \mid
\end{aligned}
$$




$$
\begin{aligned}
\leq & \left|\frac{\ell^{2}}{\Gamma(\kappa)} \int_{0}^{1}(1-\mathrm{s})^{\kappa-1}\left(\mathrm{~g}_{1}(\mathrm{~s}, \mathrm{u}(\mathrm{s}))-\mathrm{g}_{1}(\mathrm{~s}, \overline{\mathrm{u}}(\mathrm{s}))\right) d \mathrm{~s}\right| \\
& +\left|\frac{1}{\Gamma(\kappa)} \int_{0}^{\ell}(\ell-\mathrm{s})^{\kappa-1}\left(\mathcal{F}\left(\mathrm{s}, \mathrm{w}(\mathrm{s}), D^{\kappa} \mathrm{u}(\mathrm{s})\right)-\mathcal{F}\left(\mathrm{s}, \overline{\mathrm{w}}(\mathrm{s}), D^{\kappa} \overline{\mathrm{u}}(\mathrm{s})\right)\right) d \mathrm{~s}\right| \\
& +\left|\frac{\ell^{2}}{\Gamma(\kappa)} \int_{0}^{1}(1-\mathrm{s})^{\kappa-1}\left(\mathcal{F}\left(\mathrm{s}, \mathrm{w}(\mathrm{s}), D^{\kappa} \mathrm{u}(\mathrm{s})\right)-\mathcal{F}\left(\mathrm{s}, \overline{\mathrm{w}}(\mathrm{s}), D^{\kappa} \overline{\mathrm{u}}(\mathrm{s})\right)\right) d \mathrm{~s}\right| \\
\leq & \left|\frac{1}{\Gamma(\kappa+1)}\left(\mathrm{g}_{1}(\mathrm{~s}, \mathrm{u}(\mathrm{s}))-\mathrm{g}_{1}(\mathrm{~s}, \overline{\mathrm{u}}(\mathrm{s}))\right)\right| \\
& +\left|\frac{2}{\Gamma(\kappa+1)}\left(\mathcal{F}\left(\mathrm{s}, \mathrm{w}(\mathrm{s}), D^{\kappa} \mathrm{u}(\mathrm{s})\right)-\mathcal{F}\left(\mathrm{s}, \overline{\mathrm{w}}(\mathrm{s}), D^{\kappa} \overline{\mathrm{u}}(\mathrm{s})\right)\right) d \mathrm{~s}\right| \\
\leq & \left|\left(\mathrm{g}_{1}(\mathrm{~s}, \mathrm{u}(\mathrm{s}))-\mathrm{g}_{1}(\mathrm{~s}, \overline{\mathrm{u}}(\mathrm{s}))\right)\right|+2|(\mathcal{F}(\mathrm{s}, \mathrm{w}(\mathrm{s}), \omega(\mathrm{s}))-\mathcal{F}(\mathrm{s}, \overline{\mathrm{w}}(\mathrm{s}), \bar{\omega}(\mathrm{s}))) d \mathrm{~s}| \\
\leq & a_{1}\|\mathrm{u}-\overline{\mathrm{u}}\|+2\left(C_{\mathrm{g}_{1}}\|\mathrm{w}-\overline{\mathrm{w}}\|+D_{\mathrm{g}_{1}}\|\omega-\bar{\omega}\|\right) \\
\leq & a_{1}\|\mathrm{u}-\overline{\mathrm{u}}\|+2\left(C_{\mathrm{g}_{1}}\|\mathrm{w}-\overline{\mathrm{w}}\|+\frac{C_{\mathrm{g}_{1}} D_{\mathrm{g}_{1}}}{1-D_{\mathrm{g}_{1}}}\|\mathrm{w}-\overline{\mathrm{w}}\|\right) \\
= & a_{1}\|\mathrm{u}-\overline{\mathrm{u}}\|+\frac{2 C_{\mathrm{g}_{1}}}{1-D_{\mathrm{g}_{1}}}\|\mathrm{w}-\overline{\mathrm{w}}\|,
\end{aligned}
$$

which implies that

$$
\left|\mathcal{B}_{1}(\mathrm{u}, \mathrm{w})-\mathcal{B}_{1}(\overline{\mathrm{u}}, \overline{\mathrm{w}})\right| \leq C^{\prime}\|(\mathrm{u}, \mathrm{w})-(\overline{\mathrm{u}}, \overline{\mathrm{w}})\|,
$$

where $C^{\prime}=\max \left\{a_{1}, \frac{2 C_{g_{1}}}{1-D g_{1}}\right\}$, similarly

$$
\left|\mathcal{B}_{2}(\mathrm{u}, \mathrm{w})-\mathcal{B}_{2}(\overline{\mathrm{u}}, \overline{\mathrm{w}})\right| \leq D^{\prime}\|(\mathrm{u}, \mathrm{w})-(\overline{\mathrm{u}}, \overline{\mathrm{w}})\| .
$$

Now, from (3.13) and (3.14), we have

$$
\begin{aligned}
|\mathcal{B}(\mathrm{u}, \mathrm{w})-\mathcal{B}(\overline{\mathrm{u}}, \overline{\mathrm{w}})| & =\left|\mathcal{B}_{1}(\mathrm{u}, \mathrm{w})-\mathcal{B}_{1}(\overline{\mathrm{u}}, \overline{\mathrm{w}})\right|+\left|\mathcal{B}_{2}(\mathrm{u}, \mathrm{w})-\mathcal{B}_{2}(\overline{\mathrm{u}}, \overline{\mathrm{w}})\right| \\
& \leq C^{\prime}\|(\mathrm{u}, \mathrm{w})-(\overline{\mathrm{u}}, \overline{\mathrm{w}})\|+D^{\prime}\|(\mathrm{u}, \mathrm{w})-(\overline{\mathrm{u}}, \overline{\mathrm{w}})\|,
\end{aligned}
$$

it follows that

$$
|\mathcal{B}(\mathrm{u}, \mathrm{w})-\mathcal{B}(\overline{\mathrm{u}}, \overline{\mathrm{w}})| \leq\left(C^{\prime}+D^{\prime}\right)\|(\mathrm{u}, \mathrm{w})-(\overline{\mathrm{u}}, \overline{\mathrm{w}})\| .
$$

Thus, from (3.4) and (3.15), we have

$$
\begin{aligned}
|\mathcal{T}(\mathrm{u}, \mathrm{w})-\mathcal{T}(\overline{\mathrm{u}}, \overline{\mathrm{w}})| & \leq|\mathcal{A}(\mathrm{u}, \mathrm{w})-\mathcal{A}(\overline{\mathrm{u}}, \overline{\mathrm{w}})|+|\mathcal{B}(\mathrm{u}, \mathrm{w})-\mathcal{B}(\overline{\mathrm{u}}, \overline{\mathrm{w}})| \\
& \leq k_{\theta}\|(\mathrm{u}, \mathrm{w})-(\overline{\mathrm{u}}, \overline{\mathrm{w}})\|+\left(C^{\prime}+D^{\prime}\right)\|(\mathrm{u}, \mathrm{w})-(\overline{\mathrm{u}}, \overline{\mathrm{w}})\| \\
& =\left(k_{\theta}+C^{\prime}+D^{\prime}\right)\|(\mathrm{u}, \mathrm{w})-(\overline{\mathrm{u}}, \overline{\mathrm{w}})\|,
\end{aligned}
$$

it means that $\mathcal{T}$ is a contraction. Therefore system (1.1) has a unique solution. 
Example 3.1 Consider the given problem as follows:

$$
\left\{\begin{array}{l}
D^{\frac{11}{5}} \mathrm{u}(\ell)=\frac{\ell^{3}}{40}+\frac{e^{-\ell}}{50} \sin \mathrm{w}(\ell)+\frac{e^{-\ell}}{50} D^{\frac{11}{5}} \mathrm{u}(\ell), \\
D^{\frac{13}{6}} \mathrm{w}(\ell)=\frac{\ell^{2}}{50}+\frac{e^{-\pi \ell}}{30} \sin \mathrm{u}(\ell)+\frac{e^{-\pi \ell}}{30} D^{\frac{13}{6}} \mathrm{w}(\ell), \\
\mathrm{u}(0)=\frac{5}{8} \sin (\mathrm{u})+\frac{3}{4}, \quad \mathrm{u}^{\prime}(0)=1, \quad \mathrm{u}(1)=\frac{1}{\Gamma\left(\frac{11}{5}\right)} \int_{0}^{1}(1-\mathrm{s})^{\frac{6}{5}} \frac{\cos \mathrm{u}(\mathrm{s})}{30} d \mathrm{~s}, \\
\mathrm{w}(0)=\frac{2}{11} \cos (\mathrm{w})+\frac{5}{8}, \quad \mathrm{w}^{\prime}(0)=2, \quad \mathrm{w}(1)=\frac{1}{\Gamma\left(\frac{13}{6}\right)} \int_{0}^{1}(1-\mathrm{s})^{\frac{7}{6}} \frac{e^{-\mathrm{w}(\mathrm{s})}}{50} d \mathrm{~s} .
\end{array}\right.
$$

Here,

$$
\begin{aligned}
& \mathcal{F}\left(\ell, \mathrm{w}(\ell), D^{\kappa} \mathrm{u}(\ell)\right)=\frac{\ell^{3}}{40}+\frac{e^{-\ell}}{50} \sin \mathrm{w}(\ell)+\frac{e^{-\ell}}{50} D^{\frac{11}{5}} \mathrm{u}(\ell) \quad \text { and } \\
& \overline{\mathcal{F}}\left(\ell, \mathrm{u}(\ell), D^{\delta} \mathrm{w}(\ell)\right)=\frac{\ell^{2}}{50}+\frac{e^{-\pi \ell}}{30} \sin \mathrm{u}(\ell)+\frac{e^{-\pi \ell}}{30} D^{\frac{13}{6}} \mathrm{w}(\ell) .
\end{aligned}
$$

Now assumptions $\left(C_{1}\right)-\left(C_{6}\right)$ are satisfied for $k_{r}=c_{r}=\frac{5}{8}, k_{h}=c_{h}=\frac{2}{11}, M_{r}=\frac{3}{4}, M_{h}=\frac{5}{8}, z_{\mathrm{g}_{1}}=$ $a_{1}=\frac{1}{32}, z_{\mathrm{g}_{2}}=a_{2}=\frac{1}{50}, C_{\mathrm{g}_{1}}=c_{1}=\frac{1}{55}, D_{\mathrm{g}_{1}}=c_{2}=\frac{1}{65}, C_{\mathrm{g}_{2}}=d_{1}=\frac{1}{30}, D_{\mathrm{g}_{2}}=d_{2}=\frac{1}{35}, M_{\mathcal{F}}=\frac{1}{40}$, $M_{\overline{\mathcal{F}}}=\frac{1}{60}$, and $N_{\mathrm{g}_{1}}=N_{\mathrm{g}_{2}}=0$. Consider the set

$$
\mathcal{Q}=\{(\mathrm{u}, \mathrm{w}) \in C(\mathfrak{J} \times \mathfrak{R} \times \mathfrak{R}, \mathfrak{R}), \exists \varrho \in \mathfrak{J}:(\mathrm{u}, \mathrm{w})=\varrho \mathcal{T}(\mathrm{u}, \mathrm{w})\}
$$

Let $(\mathrm{u}, \mathrm{w}) \in \mathcal{Q}$ and $\varrho \in \mathfrak{J}$, then

$$
\begin{aligned}
\|(\mathrm{u}, \mathrm{w})\| & =\|\varrho \mathcal{T}(\mathrm{u}, \mathrm{w})\| \\
& =\varrho[\|\mathcal{A}(\mathrm{u}, \mathrm{w})+\mathcal{B}(\mathrm{u}, \mathrm{w})\|] \\
& \leq \varrho\left[\left(c_{\theta}+\theta\right)\|(\mathrm{u}, \mathrm{w})\|+(M+\Lambda)\right] \\
& =\varrho[0.731\|(\mathrm{u}, \mathrm{w})\|+4.375],
\end{aligned}
$$

which shows that $\mathcal{Q}$ is bounded. Thus, by Theorem 3.1, problem (3.16) possesses at least one solution, and the solution set is bounded. Further $k_{\theta}+C^{\prime}+D^{\prime} \simeq 0.762<1$, hence Theorem 3.2 guarantees that problem (3.16) has a unique solution.

Example 3.2 Consider another problem as follows:

$$
\left\{\begin{array}{l}
D^{\frac{16}{7}} \mathrm{u}(\ell)=\frac{e^{-\pi \ell}}{10+\ell^{2}}+\frac{\cos \mathrm{w}(\ell)}{52+\ell^{3}}+\frac{D^{\frac{16}{7}} \mathrm{u}(\ell)}{55+\ell^{2}}, \\
D^{\frac{9}{4}} \mathrm{w}(\ell)=\frac{e^{-30 \ell}}{35+\ell}+\frac{\cos \mathrm{u}(\ell)}{63(1+\ell)^{2}}+\frac{D^{\frac{9}{4}} \mathrm{w}(\ell)}{19+\ell^{2}}, \\
\mathrm{u}(0)=\frac{2}{25} e^{-\pi \mathrm{u}}+\frac{3}{9}, \quad \mathrm{u}^{\prime}(0)=\frac{1}{5}, \quad \mathrm{u}(1)=\frac{1}{\Gamma\left(\frac{16}{7}\right)} \int_{0}^{1}(1-\mathrm{s})^{\frac{9}{7}} \frac{\mathrm{s} \sqrt{\mathrm{u}(\mathrm{s})}}{48+\mathrm{s}} d \mathrm{~s}, \\
\mathrm{w}(0)=\frac{3}{13} \sin (\mathrm{w})+\frac{1}{18}, \quad \mathrm{w}^{\prime}(0)=\frac{2}{7}, \quad \mathrm{w}(1)=\frac{1}{\Gamma\left(\frac{9}{4}\right)} \int_{0}^{1}(1-\mathrm{s})^{\frac{5}{4}} \frac{\mathrm{s} \sqrt{\mathrm{w}(\mathrm{s})}}{75+\mathrm{s}} d \mathrm{~s} .
\end{array}\right.
$$

Here,

$$
\begin{aligned}
& \mathcal{F}\left(\ell, \mathrm{w}(\ell), D^{\kappa} \mathrm{u}(\ell)\right)=\frac{e^{-\pi \ell}}{10+\ell^{2}}+\frac{\cos \mathrm{w}(\ell)}{52+\ell^{3}}+\frac{D^{\frac{16}{7}} \mathrm{u}(\ell)}{55+\ell^{2}} \text { and } \\
& \overline{\mathcal{F}}\left(\ell, \mathrm{u}(\ell), D^{\delta} \mathrm{w}(\ell)\right)=\frac{e^{-30 \ell}}{35+\ell}+\frac{\cos \mathrm{u}(\ell)}{63(1+\ell)^{2}}+\frac{D^{\frac{9}{4}} \mathrm{w}(\ell)}{19+\ell^{2}} .
\end{aligned}
$$


Now assumptions $\left(C_{1}\right)-\left(C_{6}\right)$ are satisfied for $k_{r}=c_{r}=\frac{2}{25}, k_{h}=c_{h}=\frac{3}{13}, M_{r}=\frac{1}{3}, M_{h}=\frac{1}{18}$, $z_{\mathrm{g}_{1}}=a_{1}=\frac{1}{48}, z_{\mathrm{g}_{2}}=a_{2}=\frac{1}{75}, C_{\mathrm{g}_{1}}=c_{1}=\frac{1}{52}, D_{\mathrm{g}_{1}}=c_{2}=\frac{1}{55}, C_{\mathrm{g}_{2}}=d_{1}=\frac{1}{63}, D_{\mathrm{g}_{2}}=d_{2}=\frac{1}{19}, M_{\mathcal{F}}=$ $\frac{1}{10}, M_{\overline{\mathcal{F}}}=\frac{1}{35}$, and $N_{\mathrm{g}_{1}}=N_{\mathrm{g}_{2}}=0$. Consider the set

$$
\mathcal{Q}=\{(\mathrm{u}, \mathrm{w}) \in C(\mathfrak{J} \times \mathfrak{R} \times \mathfrak{R}, \mathfrak{R}), \exists \varrho \in \mathfrak{J}:(\mathrm{u}, \mathrm{w})=\varrho \mathcal{T}(\mathrm{u}, \mathrm{w})\}
$$

Let $(\mathrm{u}, \mathrm{w}) \in \mathcal{Q}$ and $\varrho \in \mathfrak{J}$, then

$$
\begin{aligned}
\|(\mathrm{u}, \mathrm{w})\| & =\|\varrho \mathcal{T}(\mathrm{u}, \mathrm{w})\| \\
& =\varrho[\|\mathcal{A}(\mathrm{u}, \mathrm{w})+\mathcal{B}(\mathrm{u}, \mathrm{w})\|] \\
& \leq \varrho\left[\left(c_{\theta}+\theta\right)\|(\mathrm{u}, \mathrm{w})\|+(M+\Lambda)\right] \\
& =\varrho[0.684\|(\mathrm{u}, \mathrm{w})\|+1.553]
\end{aligned}
$$

which shows that $\mathcal{Q}$ is bounded. Thus, by Theorem 3.1, problem (3.17) possesses at least one solution, and the solution set is bounded. Further $k_{\theta}+C^{\prime}+D^{\prime} \simeq 0.684<1$, hence Theorem 3.2 guarantees that problem (3.17) has a unique solution.

\section{Conclusion}

Upon the applications of a nonlinear analysis tool called degree method, we have established some appropriate results which are required for the existence and uniqueness of the solution to a coupled system of nonlinear IFDEs. Classical fixed point theory has been used to investigate the existence and uniqueness for some problems. Utilizing these results, one needs strong compact conditions due to which the area is restricted to some BVPs. Therefore we used the degree method which relaxed these conditions. There are very few results in the literature which utilized the degree method for the existence of solutions to initial and some BVPs having CFD, but a coupled system of IFDEs has not yet been investigated very well. All the results have been demonstrated by proper examples.

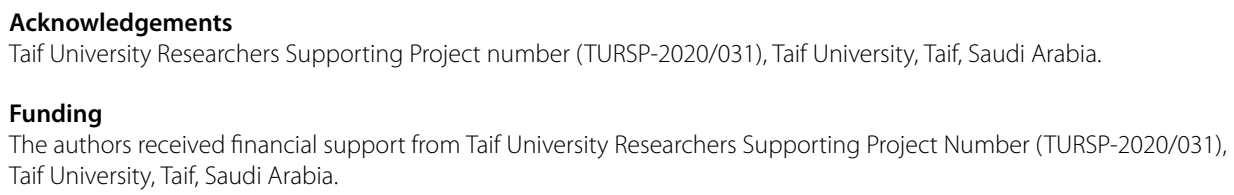

'Department of Mathematics, University of Malakand, Chakadara Dir(L), Khyber Pakhtunkhwa, Pakistan. ${ }^{2}$ Department of Basic Sciences, University of Engineering and Technology, Peshawar 25000, Pakistan. ${ }^{3}$ Section of Mathematics, International Telematic University Uninettuno, Corso Vittorio Emanuele II, 39, Roma 00186, Italy. ${ }^{4}$ Department of Mathematic, College of Science, Taif University, P.O. Box 11099, Taif 21944, Saudi Arabia.

\section{Publisher's Note}

Springer Nature remains neutral with regard to jurisdictional claims in published maps and institutional affiliations. 


\section{References}

1. Baleanu, D., Mousalou, A., Rezapour, S.: A new method for investigating approximate solutions of some fractional integro-differential equations involving the Caputo-Fabrizio derivative. Differ. Equ. Appl. 7(2) (2015)

2. Agarwal, R.P., Benchohra, M., Hamani, S.: Boundary value problems for differential inclusions with fractional order. Adv. Stud. Contemp. Math. 12(2), 181-196 (2008)

3. Ameen, R., Jarad, F., Abdeljawad, T.: Ulam stability for delay fractional differential equations with a generalized Caputo derivative. Filomat 32(15), 5265-5274 (2018)

4. Aydogan, M.S., Baleanu, D., Mousalou, A., Rezapour, S.: On high order fractional integro-differential equations including the Caputo Fabrizio derivative. Bound. Value Probl. 2018, 90 (2018)

5. Ahmad, H., Akgül, A., Khan, T.A., Stanimirović, P.S., Chu, Y.M.: New perspective on the conventional solutions of the nonlinear time-fractional partial differential equations. Complexity 2020, 8829017 (2020). https://doi.org/10.1155/2020/8829017

6. Wang, G.: Explicit iteration and unbounded solutions for fractional integral boundary value problem on an infinite interval. Appl. Math. Lett. 47, 1-7 (2015)

7. Wang, G., Pei, K., Agarwal, R., et al.: Nonlocal Hadamard fractional boundary value problem with Hadamard integral and discrete boundary conditions on a half-line. J. Comput. Appl. Math. 343, 230-239 (2018)

8. Wang, G., Ren, X.: Radial symmetry of standing waves for nonlinear fractional Laplacian Hardy-Schrödinger systems. Appl. Math. Lett. 110, 106560 (2020)

9. He, J.-H., Sun, C.: A variational principle for a thin film equation. J. Math. Chem. 57(9), 2075-2081 (2019)

10. He, J.-H.: A tutorial review on fractal spacetime and fractional calculus. Int. J. Theor. Phys. 53(11), 3698-3718 (2014)

11. Wang, K.-L., Yao, S.-W.: He's fractional derivative for the evolution equation. Therm. Sci. 24(4), 2507-2513 (2020)

12. Wang, K.-L., Liu, S.-Y.: He's fractional derivative and its application for fractional Fornberg-Whitham equation. Therm. Sci. 21(5), 2049-2055 (2017)

13. He, J.-H., Ain, Q.-T.: New promises and future challenges of fractal calculus: from two-scale thermodynamics to fractal variational principle. Therm. Sci. 24(2), 659-681 (2020)

14. Wang, J., Fečkan, M., Zhou, Y.: A survey on impulsive fractional differential equations. Fract. Calc. Appl. Anal. 19(4), 806-831 (2016)

15. Benchohra, M., Lazreg, J.E.: Exsitence and Ulam stability for nonlinear implicit fractional differential equations with Hadamard derivative. Stud. Univ. Babeş-Bolyai, Math. 62, 27-38 (2017)

16. Shah, K., Hussain, W., Thounthong, P., Borisut, P., Kumam, P., Arif, M.: On nonlinear implicit fractional differential equations with integral boundary condition involving p-Laplacian operator without compactness. Thai J. Math. 12 301-321 (2018)

17. Shah, K., Khalil, H., Khan, R.A.: Investigation of positive solution to a coupled system of impulsive boundary value problems for nonlinear fractional order differential equations. Chaos Solitons Fractals 77, 240-246 (2015)

18. Wang, G., Yang, Z., Zhang, L., Baleanu, D.: Radial solutions of a nonlinear k-Hessian system involving a nonlinear operator. Commun. Nonlinear Sci. Numer. Simul. 91, 105396 (2020)

19. Pei, K., Wang, G., Sun, Y.: Successive iterations and positive extremal solutions for a Hadamard type fractional integro-differential equations on infinite domain. Appl. Math. Comput. 312, 158-168 (2017)

20. Keten, A., Yavuz, M., Baleanu, D.: Nonlocal Cauchy problem via a fractional operator involving power kernel in Banach spaces. Fractal Fract. 3(2), 27 (2019) https://doi.org/10.3390/fractalfract3020027

21. Yavuz, M., Sulaiman, T.A., Usta, F., Bulut, H.: Analysis and numerical computations of the fractional regularized longwave equation with damping term. Math. Methods Appl. Sci. https://doi.org/10.1002/mma.6343

22. Yavuz, M., Abdeljawad, T.: Nonlinear regularized long-wave models with a new integral transformation applied to the fractional derivative with power and Mittag-Leffler kernel. Adv. Differ. Equ. 2020, Article ID 367 (2020)

23. Yavuz, M.: European option pricing models described by fractional operators with classical and generalized Mittag-Leffler kernels. Numer. Methods Partial Differ. Equ. https://doi.org/10.1002/num.22645

24. Sher, M., Shah, K., Feckan, M., Khan, R.A.: Qualitative analysis of multi-terms fractional order delay differential equations via the topological degree theory. Mathematics 8(2), 218 (2020)

25. Wang, J., Zhou, Y., Wei, W.: Study in fractional differential equations by means of topological degree methods. Numer Funct. Anal. Optim. 33(2), 216-238 (2012)

26. Ali, A., Shah, K., Li, Y.: Topological degree theory and Ulam's stability analysis of a boundary value problem of fractional differential equations. In: Front. Func. Equ. Analy. Ineq., pp. 73-92 (2019)

27. Sher, M., Shah, K., Rassias, J.: On qualitative theory of fractional order delay evolution equation via the prior estimate method. Math. Methods Appl. Sci. 43, 6464-6475 (2020)

28. Samina, Shah, K., Khan, R.A., Baleanu, D.: Study of implicit type coupled system of non-integer order differential equations with antiperiodic boundary conditions. Math. Methods Appl. Sci. 42, 2033-2042 (2019)

29. Cabada, A., Wang, G.: Positive solutions of nonlinear fractional differential equations with integral boundary value conditions. J. Math. Anal. Appl. 398(1), 403-411 (2012)

30. Isaia, F.: On a nonlinear integral equation without compactness. Acta Math. Univ. Comen. 75(2), 233-240 (2006)

31. Ali, A., Sarwar, M., Zada, M.B., Shah, K.: Degree theory and existence of positive solutions to coupled system involving proportional delay with fractional integral boundary conditions. Math. Methods Appl. Sci., 1-13 (2020)

32. Ahmad, I., Shah, K., Rahman, G., Baleanu, D.: Stability analysis for a nonlinear coupled system of fractional hybrid delay differential equations. Math. Methods Appl. Sci. 43, 8669-8682 (2020) 